\title{
Exact SER Analysis of DFrFT-Based QPSK OFDM System over Frequency Selective Rayleigh Fading Channel with CFO
}

\author{
Atul Kumar, ${ }^{1}$ Maurizio Magarini, ${ }^{1}$ Hem Dutt Joshi, ${ }^{2}$ and Rajiv Saxena ${ }^{3}$ \\ ${ }^{1}$ Dipartimento di Elettronica, Informazione e Bioingegneria, Politecnico di Milano, Italy \\ ${ }^{2}$ Department of Electronics and Communication Engineering, Thapar University, Patiala, India \\ ${ }^{3}$ Jaypee University, Anoopshahr, Uttar Pradesh, India
}

Correspondence should be addressed to Maurizio Magarini; maurizio.magarini@polimi.it

Received 30 November 2015; Revised 14 July 2016; Accepted 9 August 2016

Academic Editor: Tzonelih Hwang

Copyright (C) 2016 Atul Kumar et al. This is an open access article distributed under the Creative Commons Attribution License, which permits unrestricted use, distribution, and reproduction in any medium, provided the original work is properly cited.

\begin{abstract}
We present the exact symbol error rate (SER) expression for quadrature phase shift keying (QPSK) modulation in frequency selective Rayleigh fading channel for discrete fractional Fourier transform- (DFrFT-) based orthogonal frequency division multiplexing (OFDM) system in the presence of carrier frequency offset (CFO). The theoretical result is confirmed by means of Monte Carlo simulations. It is shown that the performance of the proposed system, at different values of the DFrFT angle parameter " $\alpha$," is better than that of OFDM based on discrete Fourier transform.
\end{abstract}

\section{Introduction}

In the recent past, discrete fractional Fourier transform (DFrFT) has found wide application as an efficient tool for performing time-frequency analysis in many fields of digital signal processing (DSP) [1]. Here, the focus is on its use in place of discrete Fourier transform (DFT) in orthogonal frequency division multiplexing (OFDM) system for wireless communications. It is well known that DFT-based OFDM system has drawn major attention in broadband wireless communication due to its various advantages like less complex equalizer, robustness against multipath fading channel, high data rate, and efficient bandwidth utilization [2]. Due to these favorable properties, OFDM has been adopted by many wireless communication standards, such as wireless local area networks, that is, IEEE 802.11a, IEEE 802.11g, and IEEE $802.11 n$, wireless metropolitan area networks, that is, IEEE802.16a, 3GPP long term evolution, and terrestrial digital video broadcasting systems $[3,4]$.

In spite of several advantages associated with OFDM, there are also some disadvantages, such as high sensitivity to carrier frequency offset (CFO) [5]. The CFO at the receiver may be introduced due to either Doppler spreading, phase noise, or mismatching of transmitter and receiver carrier frequencies $[6,7]$. The presence of CFO destroys the orthogonality between the subcarriers and generates the intercarrier interference (ICI) among them. Several methods are available in the literature to reduce the effect of ICI at the receiver like windowing [8], ICI self-cancellation [9], frequency domain equalization [10], and so forth. As shown in [11], a higher tolerance to ICI effects can be achieved by replacing DFT with DFrFT. The advantage of DFrFT over DFT was demonstrated by deriving an analytical expression of the signal-to-interference ratio (SIR) and using it to evaluate the performance. Note that, since DFrFT represents a generalization of the DFT, the analytical expression derived in [11] allows us to compute SIR for both DFT-based and DFrFT-based OFDM system.

However, in digital communication systems more interest is in the evaluation of the error probability in presence of CFO. For error probability analysis of OFDM in presence of CFO, two most widely methods are proposed in the literature. One method consists in using a Gaussian approximation for ICI, as a result of the central limit theorem, when the number of subcarriers is high [12]. However, in [13], it is shown that such an approximation leads to an overestimation of error performance, especially at high signal-to-noise ratios (SNR) values. In another method [14], a unique approach based 
on the use of characteristic functions and Beaulieu series is proposed to derive exact bit error rate (BER) expressions in the presence of ICI. By following [14], it is shown in [15] that the performance of DFrFT-based OFDM system is better than the DFT-based one in terms of BER. However, only binary phase shift keying (BPSK) modulation has been considered in [15] for BER analysis.

In this paper, we focus on performance analysis of DFrFTbased OFDM system for quadrature phase shift keying (QPSK) modulation technique in presence of CFO. The advantage of QPSK over BPSK is a well-known fact. The main contribution of this paper consists in the derivation of the analytical expression of symbol error rate (SER) for DFrFT-based QPSK OFDM system over frequency selective fading channel with CFO. With the help of theoretical and simulation results, it is shown that the performance of DFrFTbased OFDM system is better than DFT-based one in terms of SER at different values of DFrFT angle parameter " $\alpha$."

It is worth noting that the higher tolerance to CFO of DFrFT-based OFDM systems is achieved without any significant increase in implementation complexity compared to efficient fast Fourier transform (FFT) implementation of DFT. As shown in [16], when the length $N$ of the block of samples on which to compute DFrFT is a power of 2, the number of complex multiplications required is $2 \mathrm{~N}+$ $N / 2 \log _{2}(N) \approx N / 2 \log _{2}(N)$, which is of the same order as FFT. This low computational efficiency has recently increased the efforts towards the development of dedicated hardware implementations as, for example, those described in [17, 18].

The paper is organized as follows. Sections 2 and 3 introduce the system model and the mathematical derivation of the SER, respectively. Comparison of simulation and theoretical results are given in Section 4. Section 5 gives concluding remarks.

\section{System Model}

An $N$-point OFDM system is considered where the input bit stream is encoded into complex QPSK symbols that are drawn from the set $\{ \pm 1 \pm j\}$. The block of transmitted symbols applied to the input of the inverse DFrFT (IDFrFT) at discrete-time $p$ is $X_{p}(0), X_{p}(1), \ldots, X_{p}(N-1)$, where $X_{p}(k)$ is the symbol transmitted on the $k$ th subcarrier. In the following, to simplify notation, the dependence on $p$ will be omitted. After taking the $N$-point IDFrFT, the expression of the $m$ th transmitted sample is written as

$$
x(m)=\sum_{k=0}^{N-1} X(k) F_{-\alpha}(m, k), \quad m=0,1, \ldots, N-1,
$$

where $F_{-\alpha}(m, k)$ is the kernel of IDFrFT given by

$$
\begin{aligned}
& F_{-\alpha}(m, k) \\
& =\sqrt{\frac{\sin \alpha+j \cos \alpha}{N}} e^{-j m^{2} T_{s}^{2} \cot \alpha / 2} e^{-j k^{2} u^{2} \cot \alpha / 2} e^{j 2 \pi m k / N},
\end{aligned}
$$

with $T_{s}$ and $u$ being the sampling intervals in the time and fractional Fourier domain, respectively, related as $u \times T_{s}=$ $2 \pi|\sin \alpha| / N$. The fractional Fourier domain makes an angle $\alpha=a \times(\pi / 2)$ with the time-domain, where $a$ is a real number that varies from 0 to 1 . Note that, at $\alpha=\pi / 2$, that is, $a=$ 1, DFrFT converts into its DFT counterpart. By inserting a cyclic prefix (CP) of length $N_{\mathrm{CP}}$ the transmitted $m$ th sample is written as

$$
\tilde{x}(m)= \begin{cases}x(N+m), & m=-N_{\mathrm{CP}}, \ldots,-1, \\ x(m), & m=0, \ldots, N-1 .\end{cases}
$$

Considering the transmission over a frequency selective Rayleigh fading channel, the $n$th sample of the received OFDM symbol in presence of CFO $(\Delta f)$ can be expressed as

$$
r(n)=e^{j 2 \pi \epsilon n / N} \sum_{m=-N_{\mathrm{CP}}}^{N-1} \tilde{x}(m) h(n, n-m)+w(n),
$$

where $h(n, l)$ is the $l$ th coefficient of the impulse response of the multipath fading channel at time $n T_{s}, T_{u}=N T_{s}$ is the useful duration of one OFDM symbol, $\epsilon=\Delta f T_{u}=\Delta f N T_{s}$ is CFO normalized by the subcarrier spacing $1 / T_{u}$, and $w(n)$ represents the time-domain circular complex additive white Gaussian noise (AWGN) with zero mean and variance $\sigma^{2}$.

At the receiver, if the length of $\mathrm{CP}$ is higher than the maximum delay spread of the multipath channel, that is, $L \leq N_{\mathrm{CP}}$, and if the CFO is correctly estimated and compensated the received signal would be impaired only by the distortions introduced by the channel. However, because of the presence of estimation errors, time varying Doppler shift, and oscillators drift, there exists residual frequency offset and, therefore, the model in (4) holds. Hence, after removing the CP, the DFrFT of the received signal $r(n)$ can be expressed as

$$
Y(q)=\sum_{n=0}^{N-1} r(n) F_{\alpha}(q, n)
$$

where $F_{\alpha}(q, n)$ is the kernel given by

$$
\begin{aligned}
& F_{\alpha}(q, n) \\
& \quad=\sqrt{\frac{\sin \alpha-j \cos \alpha}{N}} e^{j n^{2} T_{s}^{2} \cot \alpha / 2} e^{j q^{2} u^{2} \cot \alpha / 2} e^{-j 2 \pi n q / N} .
\end{aligned}
$$

\section{SER Analysis}

Substituting the value of $r(n)$ in (5), after some mathematical manipulation the received signal on subcarrier $q$ in the presence of CFO is expressed as

$$
\begin{aligned}
Y(q)= & S(q, q) \beta(q) X(q)+\sum_{k=0, k \neq q}^{N-1} S(q, k) \beta(k) X(k) \\
& +W(q)
\end{aligned}
$$

where $X(q)$ is the complex data symbol transmitted on the $q$ th subcarrier, $\beta(q)$ is the channel frequency response for the $q$ th subcarrier, $S(q, k)$ denotes the ICI coefficient, and $W(q)$ is the 
frequency-domain circular complex AWGN with zero mean and variance $\sigma^{2}$. From (7), we denote the noiseless signal received on subcarrier $q$ as

$$
\begin{aligned}
R(q)= & S(q, q) \beta(q) X(q) \\
& +\sum_{k=0, k \neq q}^{N-1} S(q, k) \beta(k) X(k) .
\end{aligned}
$$

A block Rayleigh fading channel assumption is done, where the channel coefficients remain constant for the entire duration of the OFDM symbol. By adopting a matrix notation, let $\mathbf{h}=\left[h_{0}, h_{1}, \ldots, h_{L-1}\right]^{T}$ be the $L \times 1$ time-domain channel vector whose complex entries are i.i.d. random variables with average power $1 / L$, Rayleigh distributed amplitude, and uniform distributed phase, where $(\cdot)^{T}$ denotes the transpose operation. The vector containing the frequency domain complex channel coefficients for all the subcarriers can be expressed as $[\beta(0), \beta(1), \ldots, \beta(N-1)]^{T}=\mathscr{F}_{L} \mathbf{h}$, where $\mathscr{F}_{L}$ is $N \times L$ matrix obtained by taking columns $1: L$ of the $N \times N$ DFrFT matrix defined in [11]. The ICI coefficient is defined in [15] as

$$
\begin{aligned}
S(q, k)= & \frac{1}{N} \sum_{n=0}^{N-1} e^{j(2 \pi \epsilon n / N)} \sum_{m=0}^{N-1} e^{-j\left(\left(m^{2}-n^{2}\right) T_{s}^{2} \cot \alpha / 2\right)} \\
& \times e^{j\left(\left(q^{2}-k^{2}\right) u^{2} \cot \alpha / 2\right)} \times e^{j(2 \pi(m k-n q) / N)} .
\end{aligned}
$$

The equalized signal on the first subcarrier is

$$
\begin{aligned}
\bar{\beta}(0) Y(0)= & S(0,0)|\beta(0)|^{2} X(0) \\
& +\sum_{k=1}^{N-1} S(0, k) \bar{\beta}(0) \beta(k) X(k) \\
& +\bar{\beta}(0) W(0),
\end{aligned}
$$

where $\bar{\beta}(0)$ is the complex conjugate of $\beta(0)$. Without loosing generality, in what follows we assume that symbol transmitted on the first subcarrier is $X(0)=1+j$. Therefore, considering the complex plane, the probability of making a correct decision corresponds to the probability that $\bar{\beta}(0) Y(0) \mid X(0)=$ $1+j$ falls in the first quadrant $D_{1}$. Using standard procedure, the probability of making a correct decision conditioned on the given value of $\bar{\beta}(0) R(0)$ is

$$
\begin{aligned}
P_{c}\left(\bar{\beta}(0) Y(0) \in D_{1} \mid X(0)=1+j, \bar{\beta}(0) R(0)\right) \\
=Q\left(-\frac{\Re[\bar{\beta}(0) R(0)]}{\sigma_{\beta}}\right) \\
\quad \times Q\left(-\frac{\Im[\bar{\beta}(0) R(0)]}{\sigma_{\beta}}\right),
\end{aligned}
$$

where $\mathfrak{R}[c](\mathfrak{I}[c])$ denotes the real (imaginary) part of complex number $c$ and $\sigma_{\beta}=|\beta(0)| \sigma$. The probability of correct decision is obtained by averaging (11) over the joint two-dimensional (2D) probability density function (PDF) defined by the real and imaginary parts of $\bar{\beta}(0) R(0)$ as

$$
\begin{aligned}
P_{c} & \left(\bar{\beta}(0) Y(0) \in D_{1} \mid X(0)=1+j\right) \\
\quad & \iint_{-\infty}^{\infty} P_{c}\left(\bar{\beta}(0) Y(0) \in D_{1} \mid X(0)=1\right. \\
& +j, \mathfrak{R}[\bar{\beta}(0) R(0)], \mathfrak{\Im}[\bar{\beta}(0) R(0)]) \\
& \cdot p(\mathfrak{R}[\bar{\beta}(0) R(0)], \mathfrak{\Im}[\bar{\beta}(0) R(0)] \mid X(0)=1 \\
& +j) \cdot d \mathfrak{R}[\bar{\beta}(0) R(0)] d \mathfrak{\Im}[\bar{\beta}(0) R(0)] .
\end{aligned}
$$

As shown in [19], the simplest way to derive a closed form expression of $p(\mathfrak{R}[\bar{\beta}(0) R(0)], \mathfrak{I}[\bar{\beta}(0) R(0)] \mid X(0)=1+j)$ is to compute its $2 \mathrm{D}$ characteristic function $(\mathrm{CHF})$ in the frequency domain. Following the mathematical derivation reported in [20], the resulting $2 \mathrm{D} \mathrm{CHF}$ is

$$
\begin{aligned}
& \psi\left(\omega_{I}, \omega_{\mathrm{Q}}\right) \\
& =e^{j|\beta(0)|^{2}\left(\omega_{I}(\Re[S(0,0)]-\Im[S(0,0)])+\omega_{\mathrm{Q}}(\mathfrak{\Im}[S(0,0)]+\Re[S(0,0)])\right)} \\
& \quad \times \prod_{k=1}^{N-1} \cos \left(\omega_{I} \mathfrak{R}[S(0, k) \bar{\beta}(0) \beta(k)]\right. \\
& \left.+\omega_{\mathrm{Q}} \mathfrak{\Im}[S(0, k) \bar{\beta}(0) \beta(k)]\right) \\
& \quad \times \cos \left(\omega_{I} \mathfrak{\Im}[S(0, k) \bar{\beta}(0) \beta(k)]\right. \\
& \left.\quad-\omega_{\mathrm{Q}} \mathfrak{R}[S(0, k) \bar{\beta}(0) \beta(k)]\right) .
\end{aligned}
$$

The 2D PDF resulting from the inverse Fourier transform of (13) is

$$
\begin{aligned}
& p(\Re[\bar{\beta}(0) R(0)], \mathfrak{\Im}[\bar{\beta}(0) R(0)] \mid X(0)=1+j)=\frac{1}{2^{2 N-2}} \\
& \quad \cdot \sum_{k=1}^{2^{N-2}} \sum_{n=1}^{N-2} \sum_{m=1}^{4} \delta\left[\Re(\bar{\beta}(0) R(0))-\left(|\beta(0)|^{2} D_{A}+\varphi_{k, n}[1, m]\right)\right] \\
& \quad \times \delta\left[\mathfrak{I}(\bar{\beta}(0) R(0))-\left(|\beta(0)|^{2} D_{B}+\varphi_{k, n}[2, m]\right)\right] .
\end{aligned}
$$

In the above equation $\delta[\cdot]$ is the Dirac delta function, $D_{A}=$ $\mathfrak{R}[S(0,0)]-\mathfrak{J}[S(0,0)], D_{B}=\mathfrak{R}[S(0,0)]+\mathfrak{J}[S(0,0)]$, and $\varphi_{k, n}[p, q]$ is the entry $(p, q)$ of the $2 \times 4$ matrix $\mathbf{G}=$

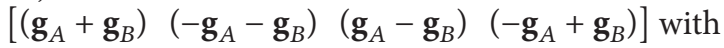

$$
\begin{aligned}
& \mathbf{g}_{A}=\left[\mathfrak{R}\left[\bar{\beta}(0) \mathbf{e}_{k}^{T} \boldsymbol{\Lambda} \boldsymbol{\beta}\right] \mathfrak{\Im}\left[\bar{\beta}(0) \mathbf{e}_{n}^{T} \boldsymbol{\Lambda} \boldsymbol{\beta}\right]\right]^{T}, \\
& \mathbf{g}_{B}=\left[\mathfrak{I}\left[\bar{\beta}(0) \mathbf{e}_{k}^{T} \boldsymbol{\Lambda} \boldsymbol{\beta}\right]-\mathfrak{R}\left[\bar{\beta}(0) \mathbf{e}_{n}^{T} \boldsymbol{\Lambda} \boldsymbol{\beta}\right]\right]^{T},
\end{aligned}
$$

where $\boldsymbol{\beta}=[\beta(1), \beta(2), \ldots, \beta(N-1)]^{T}, \boldsymbol{\Lambda}=\operatorname{diag}(S(0,1)$, $S(0,2), \ldots, S(0, N-1))$, and $\mathbf{e}_{k}$ is an $N \times 1$ vector corresponding to the binary codeword of the number $2^{N-1}-k$, in which zeros have been substituted with -1 s. Note that, because of the symmetry of the QPSK constellation, in what follows the conditioning on $X(0)=1+j$ will be omitted for keeping notation simple. 
It is worth noting that the $2 \mathrm{D}$ PDF given in (14) is a function of $(\beta(0), \boldsymbol{\beta})$ and, therefore, by replacing it in (12) and averaging we get

$$
\begin{aligned}
P_{c}\left(\bar{\beta}(0) Y(0) \in D_{1} \mid \beta(0), \boldsymbol{\beta}\right) \\
=\frac{1}{2^{2 N-2}} \sum_{k=1}^{2^{N-2} 2^{N-2}} \sum_{n=1}^{4} \sum_{m=1}^{4} Q\left(-\frac{|\beta(0)|^{2} S_{A}+\varphi_{k, n}[1, m]}{\sigma_{\beta}}\right) \\
\quad \times Q\left(-\frac{|\beta(0)|^{2} S_{B}+\varphi_{k, n}[2, m]}{\sigma_{\beta}}\right)
\end{aligned}
$$

where the dependence on $(\beta(0), \boldsymbol{\beta})$ is implicit in $\varphi_{k, n}[1, m]$ and $\varphi_{k, n}[2, m]$. The analytical expression of the SER given $(\beta(0), \boldsymbol{\beta})$ is

$$
\begin{aligned}
P_{s}(\xi \mid \beta(0), \boldsymbol{\beta}) \stackrel{\Delta}{=} & P_{s}\left(\bar{\beta}(0) Y(0) \notin D_{1} \mid \beta(0), \boldsymbol{\beta}\right) \\
= & 1 \\
& \quad-P_{c}\left(\bar{\beta}(0) Y(0) \in D_{1} \mid \beta(0), \boldsymbol{\beta}\right) .
\end{aligned}
$$

The analytic expression of the unconditional SER is obtained from

$$
\begin{aligned}
P_{s}(\xi)= & \int_{\beta(0)} \int_{\beta} P_{s}(\xi \mid \beta(0), \boldsymbol{\beta}) P_{\beta \mid \beta(0)} \\
& \cdot(\boldsymbol{\beta} \mid \beta(0)) d \beta p_{\beta(0)}(\beta(0)) d \beta(0) .
\end{aligned}
$$

It is worth noting that the derivation of $P_{s}(\xi)$ in (18) involves the computation of an $N$-dimensional integral and, therefore, it is difficult to evaluate. The computation can be simplified by observing that, for the considered frequency selective Rayleigh fading channel model, the entries of the frequency domain channel vector $\boldsymbol{\beta}$ are a mixture of complex Gaussian random variables. As a consequence, for a given value of $\beta(0)$, the two random variables $\varphi_{k, n}[1, m]$ and $\varphi_{k, n}[2, m]$ appearing in (16) are Gaussian and they can be fully specified through their mean and variance. The analytical expression of $P_{s}(\xi \mid \beta(0))$ is obtained by using the conditional mean $|\beta(0)|^{2} \vartheta_{k, n}[i, m] \mid \beta(0)$ and the conditional variance $|\beta(0)|^{2} v_{k, n}[m] / 2 \mid \beta(0)$ in the Gaussian PDFs of $\varphi_{k, n}[i, m] \mid$ $\beta(0), i=1,2$, that are used to average (17). Following the mathematical derivation of [20], the conditional probability of error after the average is

$$
\begin{aligned}
& P_{s}(\xi \mid \beta(0)) \\
& =1 \\
& -\frac{1}{2^{2 N-2}} \sum_{k=1}^{2^{N-2} 2^{N-2}} \sum_{n=1}^{4} \sum_{m=1}^{4} Q\left(-\frac{|\beta(0)| S_{A}+\vartheta_{k, n}[1, m]}{\sigma \sqrt{1+\nu_{k, n}[m] / 2 \sigma^{2}}}\right) \\
& \times Q\left(-\frac{|\beta(0)| S_{B}+\vartheta_{k, n}[2, m]}{\sigma \sqrt{1+v_{k, n}[m] / 2 \sigma^{2}}}\right),
\end{aligned}
$$

where

(i) $\vartheta_{k, n}[i, m]$ is the entry $(i, m)$ of the $2 \times 4$ matrix $\mathbf{W}=$ $\left[\begin{array}{llll}\left(\mathbf{w}_{A}+\mathbf{w}_{B}\right) & \left(-\mathbf{w}_{A}-\mathbf{w}_{B}\right) & \left(\mathbf{w}_{A}-\mathbf{w}_{B}\right) & \left(-\mathbf{w}_{A}+\mathbf{w}_{B}\right)\end{array}\right]$ with

$$
\begin{aligned}
& \mathbf{w}_{A}=C_{\beta(0) \beta(0)}^{-1}\left[\mathfrak{R}\left[\mathbf{e}_{k}^{T} \boldsymbol{\Lambda} \mathbf{C}_{\boldsymbol{\beta} \beta(0)}\right] \mathfrak{J}\left[\mathbf{e}_{k}^{T} \boldsymbol{\Lambda} \mathbf{C}_{\boldsymbol{\beta} \beta(0)}\right]\right]^{T} \text {, } \\
& \mathbf{w}_{B}=C_{\beta(0) \beta(0)}^{-1}\left[\mathfrak{I}\left[\mathbf{e}_{n}^{T} \boldsymbol{\Lambda} \mathbf{C}_{\boldsymbol{\beta} \beta(0)}\right]-\mathfrak{R}\left[\mathbf{e}_{n}^{T} \boldsymbol{\Lambda} \mathbf{C}_{\boldsymbol{\beta} \beta(0)}\right]\right]^{T},
\end{aligned}
$$

$\mathbf{C}_{\beta \mid \beta(0)}$ being the channel autocovariance matrix given in [15];

(ii) $v_{k, n}[m]$ is the $m$ th element of the $1 \times 4$ vector $\nu=$ $\left[\begin{array}{llll}\nu_{1} & v_{2} & v_{3} & v_{4}\end{array}\right]$, where $\nu_{i}=\boldsymbol{\zeta}_{i}^{T} \Lambda \mathbf{C}_{\beta \beta(0)} \Lambda \boldsymbol{\zeta}_{i}$ and $\boldsymbol{\zeta}_{1}=$ $\mathbf{e}_{k}+\mathbf{e}_{n}, \zeta_{2}=-\mathbf{e}_{k}-\mathbf{e}_{n}, \zeta_{3}=\mathbf{e}_{k}-\mathbf{e}_{n}, \zeta_{4}=-\mathbf{e}_{k}+\mathbf{e}_{n}$.

The SER expression is obtained by averaging over the PDF of $\beta(0)$ as [21]

$$
P_{s}(\xi)=\int_{\beta(0)} P_{s}(\xi \mid \beta(0)) p_{\beta(0)}(\beta(0)) d \beta(0) .
$$

Considering that $p_{\beta(0)}(\beta(0))$ is Rayleigh distributed, the SER expression for QPSK resulting from computation of (21) is given by

$$
\begin{aligned}
& P_{s}(\xi)=\frac{3}{4}-\frac{1}{2^{2 N-1}} \\
& \cdot \sum_{k=1}^{2^{N-2}} \sum_{n=1}^{2^{N-2}} \sum_{m=1}^{4} \sqrt{\frac{C_{\beta(0) \beta(0)} \varrho}{2+\varrho \nu_{k, n}[m]+C_{\beta(0) \beta(0)} \varrho \Psi_{k, n}^{2}[1, m]}} \\
& \times \Psi_{k, n}[1, m]\left(\frac{1}{2}+\frac{1}{\pi}\right. \\
& \left.\cdot \operatorname{atan}\left\{\sqrt{\frac{C_{\beta(0) \beta(0)} \varrho \Psi_{k, n}^{2}[2, m]}{2+\varrho v_{k, n}[m]+C_{\beta(0) \beta(0)} \varrho \Psi_{k, n}^{2}[1, m]}}\right\}\right) \\
& +\sqrt{\frac{C_{\beta(0) \beta(0)} \varrho}{2+\varrho v_{k, n}[m]+C_{\beta(0) \beta(0)} \varrho^{2}{ }_{k, n}[2, m]}} \times \Psi_{k, n}[2, \\
& m]\left(\frac{1}{2}+\frac{1}{\pi}\right. \\
& \cdot \operatorname{atan}\left\{\sqrt{\frac{C_{\beta(0) \beta(0)} \varrho \Psi_{k, n}^{2}[1, m]}{2+\varrho v_{k, n}[m]+C_{\beta(0) \beta(0)} \varrho^{2}{ }_{k, n}[2, m]}}\right\},
\end{aligned}
$$

where $\varrho=E_{s} / N_{0}$ is the SNR, with $E_{s}$ and $N_{0}$ denoting the average energy per symbol and the power spectral density of complex AWGN, respectively, and $\Psi_{k, n}[i, m]$ is the entry $(i, m)$ of the $2 \times 4$ matrix $\boldsymbol{\Phi}=\mathbf{D} \otimes \mathbf{1}_{1 \times 4}+\mathbf{W}$, in which $\otimes$ is the Kronecker product, $\mathbf{1}_{1 \times 4}$ is $1 \times 4$ vectors of $\mathbf{1 s}$, and $\mathbf{D}=\left[\begin{array}{ll}D_{A} & D_{B}\end{array}\right]^{T}$. Substituting $\alpha=\pi / 2$ in $S(q, k)$ and $C_{\beta(0) \beta(0)}=2 \sigma^{2}$ in (22) we get the SER expression of QPSK for conventional OFDM system given by equation (16) of [5]. This is in conformity with the fact that DFrFT-based OFDM is a generalization of conventional DFT-based OFDM system. 


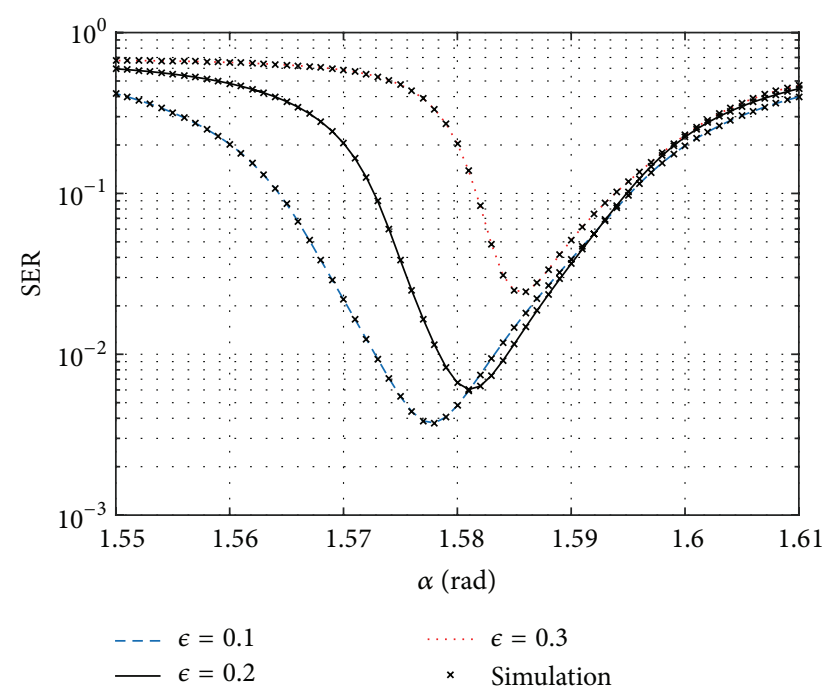

FIGURE 1: Simulated and computed SER versus angle parameter " $\alpha$ " for different values of the normalized CFO $\epsilon$ in case of 16point DFrFT-OFDM QPSK transmission over a 2-tap equal power delay profile frequency selective Rayleigh fading channel at $E_{s} / N_{0}=$ $25 \mathrm{~dB}$.

\section{Simulation Results}

First of all, in order to verify the correctness of the analysis done in Section 3, SER results obtained from Monte Carlo (MC) simulation, marked as $\times$, are compared to those predicted by (22). The impact of DFrFT angle parameter $\alpha$ on the performance is evaluated to find the value that minimizes the SER for different values of the normalized CFO $\epsilon$ at a given SNR. Analytical and MC simulation results obtained for $\epsilon=0.1,0.2$ and 0.3 at $E_{s} / N_{0}=25 \mathrm{~dB}$ with $N=16$ in case of transmission over a frequency selective Rayleigh fading channel with $L=2$ are shown in Figure 1. A perfect match is observed between simulation and analytical results. The choice of $E_{s} / N_{0}=25 \mathrm{~dB}$ allows having more pronounced minima and, at the same time, represents a value that is about in the middle of the range of SNR values considered for measuring SER that will be shown in Figures 2, 3, 4 and 5. Even if we have not reported this in the present results, for $L=5$ the same values of optimal $\alpha$ have been found for the same values of $\epsilon, E_{s} / N_{0}$, and $N$. It is worth noting that Figure 1 shows results only for positive values of $\epsilon$. For negative values $-|\epsilon|$, with $|\cdot|$ denoting the modulus, the resulting optimal value $\alpha_{\mathrm{opt}}^{(-)}$is specular, with respect to $\pi / 2$, to the optimal $\alpha_{\mathrm{opt}}^{(+)}$ obtained for a positive normalized CFO value with the same absolute value $|\epsilon|$; that is, $\alpha_{\mathrm{opt}}^{(-)}=\pi / 2-\alpha_{\mathrm{opt}}^{(+)}$.

From Figure 1 we observe that the optimal value of $\alpha$ is not the same for all the considered values of $\epsilon$. The sensitivity of the SER performance to variations of $\epsilon$ can be evaluated if we consider that in practical situations the normalized $\mathrm{CFO}$ is not a constant parameter but it has small random deviations $\Delta \epsilon$ around a fixed value $\epsilon$. As in [22], we assume that $\Delta \epsilon$ can be modeled as a zero mean Gaussian random variable. Figure 2 reports the SER performance versus the angle parameter $\alpha$ for $\epsilon=0.2, E_{s} / N_{0}=25 \mathrm{~dB}$, and $\sigma_{\Delta \epsilon} \in\{0,2$.

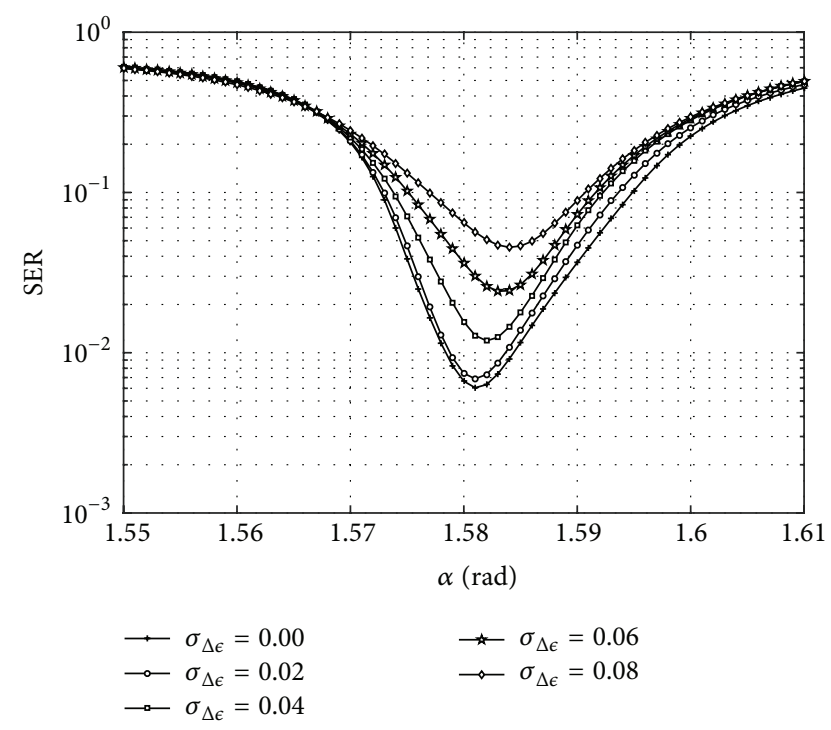

FIGURE 2: Computed SER versus angle parameter " $\alpha$ " for $\epsilon=$ 0.2 , with different values of $\sigma_{\Delta \epsilon}$, in case of 16-point DFrFT-OFDM QPSK transmission over a 2-tap equal power delay profile frequency selective Rayleigh fading channel at $E_{s} / N_{0}=25 \mathrm{~dB}$.

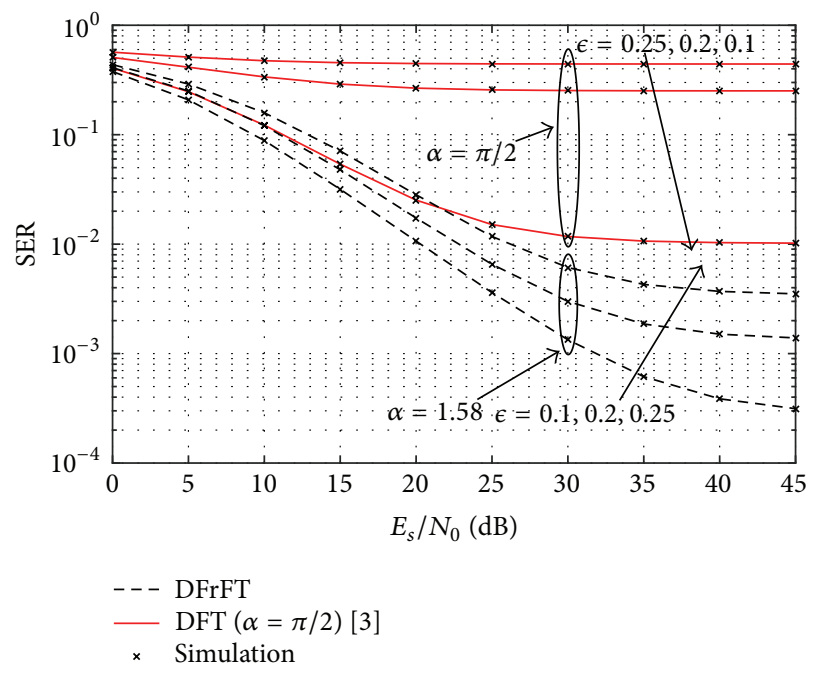

FIGURE 3: Comparison of SER between 16-point DFT- and DFrFTbased OFDM QPSK transmission over a 2-tap equal power delay profile frequency selective Rayleigh fading channel at different values of the normalized CFO $\epsilon$.

$\left.10^{-2}, \ldots, 8 \cdot 10^{-2}\right\}$, with $\sigma_{\Delta \epsilon}$ being the standard deviation of $\Delta \epsilon$. Numerical results shown in Figure 4 have been obtained by averaging the performance given by (22), computed at different values of $\epsilon+\Delta \epsilon$ for a given $\alpha$ and SNR, with the discretized Gaussian PDF of $\Delta \epsilon$ for the considered $\sigma_{\Delta \epsilon}$. Discretization of the Gaussian PDF has been done by dividing the interval $\left(-3 \sigma_{\Delta \epsilon}, 3 \sigma_{\Delta \epsilon}\right)$ in bins of size $10^{-2}$ and normalizing the resulting discrete probability to add up to 1 . Computation of (22) has been done considering as values of $\epsilon+\Delta \epsilon$ the centers of the discrete intervals. From Figure 2 we observe that when $\sigma_{\Delta \epsilon}$ increases from 0 to 0.08 the optimal value of $\alpha$ 


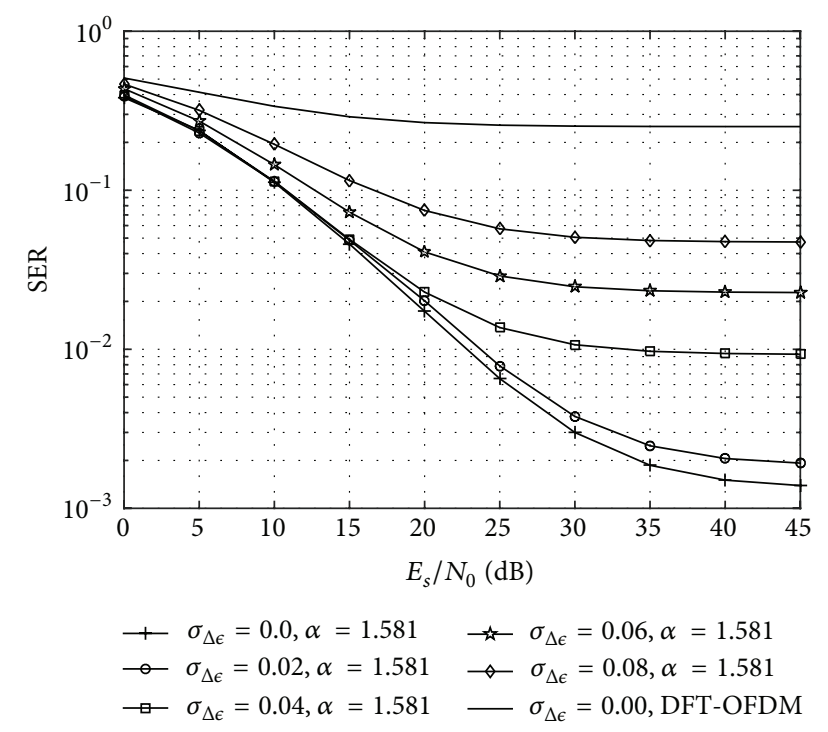

FIGURE 4: SER versus SNR for 16-point DFrFT-based OFDM QPSK transmission over a 2-tap equal power delay profile frequency selective Rayleigh fading channel with $\epsilon=0.2$ at different values of the normalized CFO standard deviation $\sigma_{\Delta \epsilon}$.

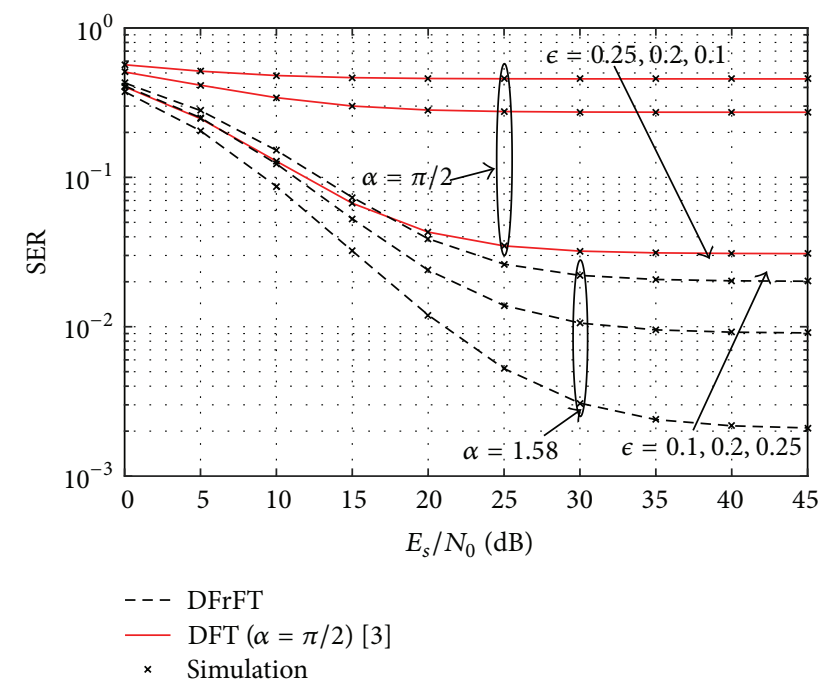

FIGURE 5: Comparison of SER between 16-point DFT- and DFrFTbased OFDM QPSK transmission over a 5-tap equal power delay profile frequency selective Rayleigh fading channel at different values of the normalized $\mathrm{CFO} \epsilon$.

has a slight increase that the corresponding SER performance starts having a significant degradation when $\sigma_{\Delta \epsilon}>0.02$. Similar results have been obtained for $\epsilon=0.1$ and $\epsilon=0.3$, not reported here.

In order to analyze the SER versus $E_{s} / N_{0}$ of DFrFT-based OFDM system for $\epsilon=0.1,0.2,0.25$, we have considered the value of $\alpha=1.58$ that as shown in Figure 1 allows achieving a good performance for all the considered values of $\epsilon$. A comparison between the performance of DFrFT-based OFDM system and that based on DFT, obtained by setting $\alpha=\pi / 2$, in (22), is shown in Figure 3. From the presented results we observe that DFrFT allows achieving a significant improvement of SER performance over DFT, especially in the high SNR region, where the error floor is reduced by more than an order of magnitude.

For the DFrFT-based OFDM system the sensitivity of SER performance to random deviations from $\epsilon=0.2$ is studied in Figure 4 considering the same values $\sigma_{\Delta \epsilon}$ of Figure 2. In our simulations, for all the values of $\sigma_{\Delta \epsilon}$, we have chosen $\alpha=1.581$ that, from Figure 2, gives a minimum SER at $\epsilon=0.2$ when $\sigma_{\Delta \epsilon}=0$. This allows us to study the impact of $\sigma_{\Delta \epsilon}$ on the performance when the design of $\alpha$ is done considering the normalized $\mathrm{CFO} \epsilon$ as fixed constant parameter. From Figure 4 we see that for $\sigma_{\Delta \epsilon}>0.02$ there is a substantial performance degradation in comparison to $\sigma_{\Delta \epsilon}=0$. Note that at high SNR the error floor for $\sigma_{\Delta \epsilon}=0.08$ increases up to more than one order of magnitude with respect to $\sigma_{\Delta \epsilon}=0$. However, by mean of a comparison with the performance achieved by DFT-based OFDM with $\epsilon=0.2$ and $\sigma_{\Delta \epsilon}=0$, reported in Figure 4 as a reference, we see that DFrFT-based OFDM always provides a better performance for all the considered values of $\sigma_{\Delta \epsilon}$.

Figure 5 reports SER versus $E_{s} / N_{0}$ of a DFrFT-based OFDM system for $\epsilon=0.1,0.2,0.25$, and $N=16$ in case of transmission over a frequency selective Rayleigh fading channel with $L=5$. The same value $\alpha=1.58$, considered for $L=2$, is here used for $L=5$. Again, at high SNR the DFrFT-based OFDM system allows achieving a gain of more than one order of magnitude over DFT-based OFDM system in SER performance.

\section{Conclusion}

This article provides an exact SER expression of QPSK modulation technique for DFrFT-based OFDM system over frequency selective Rayleigh fading channel in presence of CFO. The results produced are clearly demonstrating that the proposed DFrFT-based OFDM system overcomes the DFTbased one. A lower SER can be achieved by properly choosing the DFrFT angle parameter " $\alpha$." The sensitivity of the SER performance to random deviations from the fixed value of CFO used for the design of the optimal " $\alpha$ " is also presented. The study confirms that the DFrFT system prevails due to the controlled SER through the angle parameter " $\alpha$." It is worth noting that this improvement is achieved without adding any implementation complexity cost compared to DFT-based OFDM.

\section{Competing Interests}

The authors declare that they have no competing interests.

\section{References}

[1] E. Sejdić, I. Djurović, and L. J. Stanković, "Fractional Fourier transform as a signal processing tool: an overview of recent developments," Signal Processing, vol. 91, no. 6, pp. 13512-1369, 2011.

[2] G. L. Stüber, J. R. Barry, S. W. Mclaughlin, Y. E. Li, M. A. Ingram, and T. G. Pratt, "Broadband MIMO-OFDM wireless 
communications," Proceedings of the IEEE, vol. 92, no. 2, pp. 271293, 2004.

[3] B. Bellalta, L. Bononi, R. Bruno, and A. Kassler, "Next generation IEEE 802.11 Wireless Local Area Networks: Current status, future directions and open challenges," Computer Communications, vol. 75, pp. 1-25, 2016.

[4] C. Shahriar, M. La Pan, M. Lichtman et al., "PHY-layer resiliency in OFDM communications: a tutorial," IEEE Communications Surveys \& Tutorials, vol. 17, no. 1, pp. 292-314, 2015.

[5] A. M. Hamza and J. W. Mark, "Closed form SER expressions for QPSK OFDM systems with frequency offset in Rayleigh fading channels," IEEE Communications Letters, vol. 18, no. 10, pp. 1687-1690, 2014.

[6] L. Barletta, F. Bergamelli, M. Magarini, N. Carapellese, and A. Spalvieri, "Pilot-aided trellis-based demodulation," IEEE Photonics Technology Letters, vol. 25, no. 13, pp. 1234-1237, 2013.

[7] L. Barletta, M. Magarini, S. Pecorino, and A. Spalvieri, "Upper and lower bounds to the information rate transferred through first-order Markov channels with free-running continuous state," Institute of Electrical and Electronics Engineers. Transactions on Information Theory, vol. 60, no. 7, pp. 3834-3844, 2014.

[8] M. Ma, B. Jiao, and W. C. Y. Lee, "A dual-window technique for enhancing robustness of OFDM against frequency offset," IEEE Communications Letters, vol. 12, no. 1, pp. 17-19, 2008.

[9] M.-X. Chang, "A novel algorithm of inter-subchannel interference self-cancellation for OFDM systems," IEEE Transactions on Wireless Communications, vol. 6, no. 8, pp. 2881-2893, 2007.

[10] M. Magarini, L. Barletta, and A. Spalvieri, "Efficient computation of the feedback filter for the hybrid decision feedback equalizer in highly dispersive channels," IEEE Transactions on Wireless Communications, vol. 11, no. 6, pp. 2245-2253, 2012.

[11] J. Zheng and Z. Wang, "ICI analysis for FRFT-OFDM systems to frequency offset in time-frequency selective fading channels," IEEE Communications Letters, vol. 14, no. 10, pp. 888-890, 2010.

[12] L. Rugini and P. Banelli, "BER of OFDM systems impaired by carrier frequency offset in multipath fading channels," IEEE Transactions on Wireless Communications, vol. 4, no. 5, pp. 2279-2288, 2005.

[13] T. Keller and L. Hanzo, "Adaptive multicarrier modulation: a convenient framework for time-freqyebcy processing in wireless communication," Proceedings of the IEEE, vol. 88, no. 5, pp. 611-640, 2000.

[14] K. Sathananthan and C. Tellambura, "Probability of error calculation of OFDM systems with frequency offset," IEEE Transactions on Communications, vol. 49, no. 11, pp. 1884-1888, 2001.

[15] S. Kumari, S. K. Rai, A. Kumar, H. D. Joshi, A. K. Singh, and R. Saxena, "Exact BER analysis of FRFT-OFDM system over frequency selective Rayleigh fading channel with CFO," Electronics Letters, vol. 49, no. 20, pp. 1299-1301, 2013.

[16] A. K. Singh and R. Saxena, "DFRFT: a classified review of recent methods with its application," Journal of Engineering, vol. 2013, Article ID 214650, 13 pages, 2013.

[17] K. K. Sharma and A. Samad, "Application specific integrated circuit implementation of discrete fractional fourier transform," International Journal of Information and Electronics Engineering, vol. 3, no. 5, pp. 444-447, 2013.

[18] Q. Zou, L. Li, Q. Huang, and F. Wang, "Implementation of weighted summation type fractional Fourier transform on FPGA," in Seventh International Conference on Digital Image Processing (ICDIP '15), vol. 9631 of Proceedings of SPIE, Los Angeles, Calif, USA, July 2015.
[19] A. Annamalai, C. Tellambura, and V. K. Bhargava, "A general method for calculating error probabilities over fading channels," IEEE Transactions on Communications, vol. 53, no. 5, pp. 841852, 2005.

[20] P. Dharmawansa, N. Rajatheva, and H. Minn, "An exact error probability analysis of OFDM systems with frequency offset," IEEE Transactions on Communications, vol. 57, no. 1, pp. 26-31, 2009.

[21] M. K. Simon and M.-S. Alouini, Digital Communication over Fading Channels, Hoboken, NJ, USA, Wiley-Interscience, 2nd edition, 2005.

[22] A. J. Coulson, "Maximum likelihood synchronization for OFDM using a pilot symbol: analysis," IEEE Journal on Selected Areas in Communications, vol. 19, no. 12, pp. 2495-2503, 2001. 


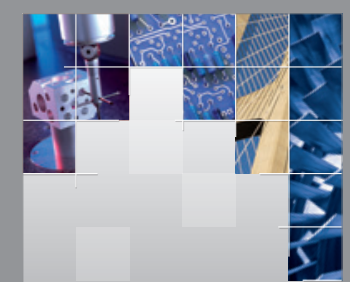

\section{Enfincering}
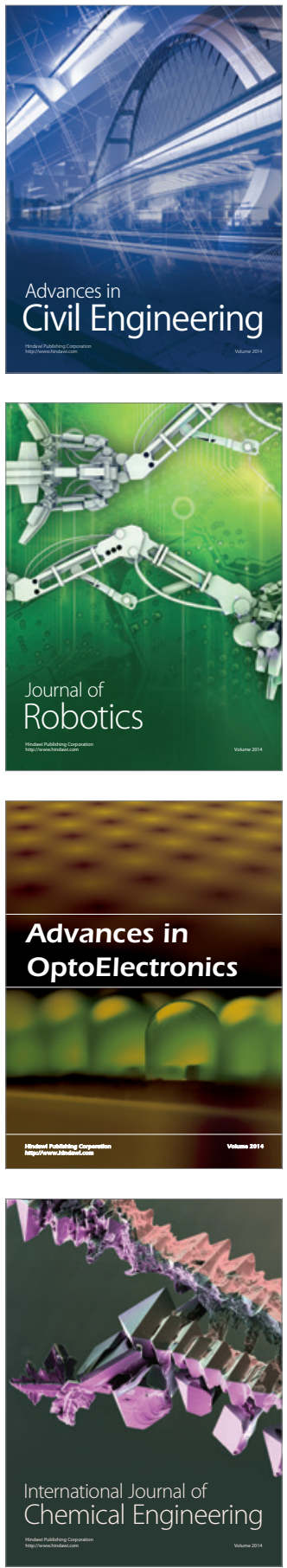

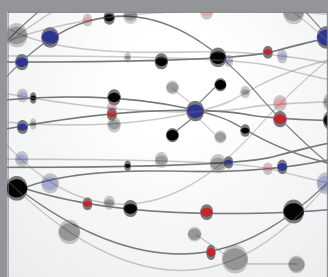

The Scientific World Journal

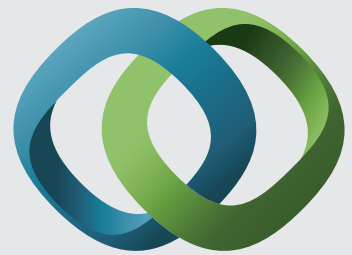

\section{Hindawi}

Submit your manuscripts at

http://www.hindawi.com
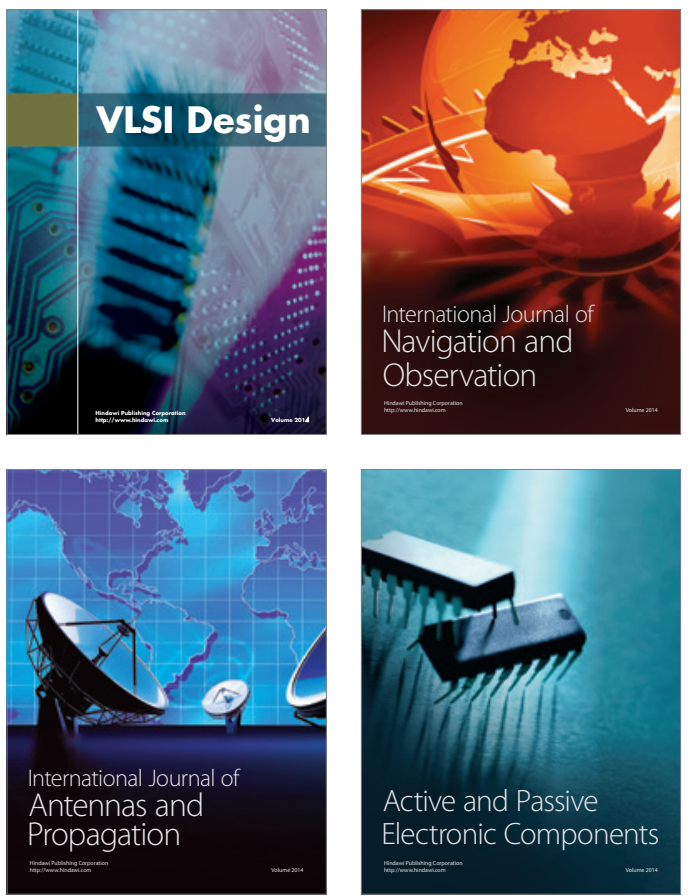
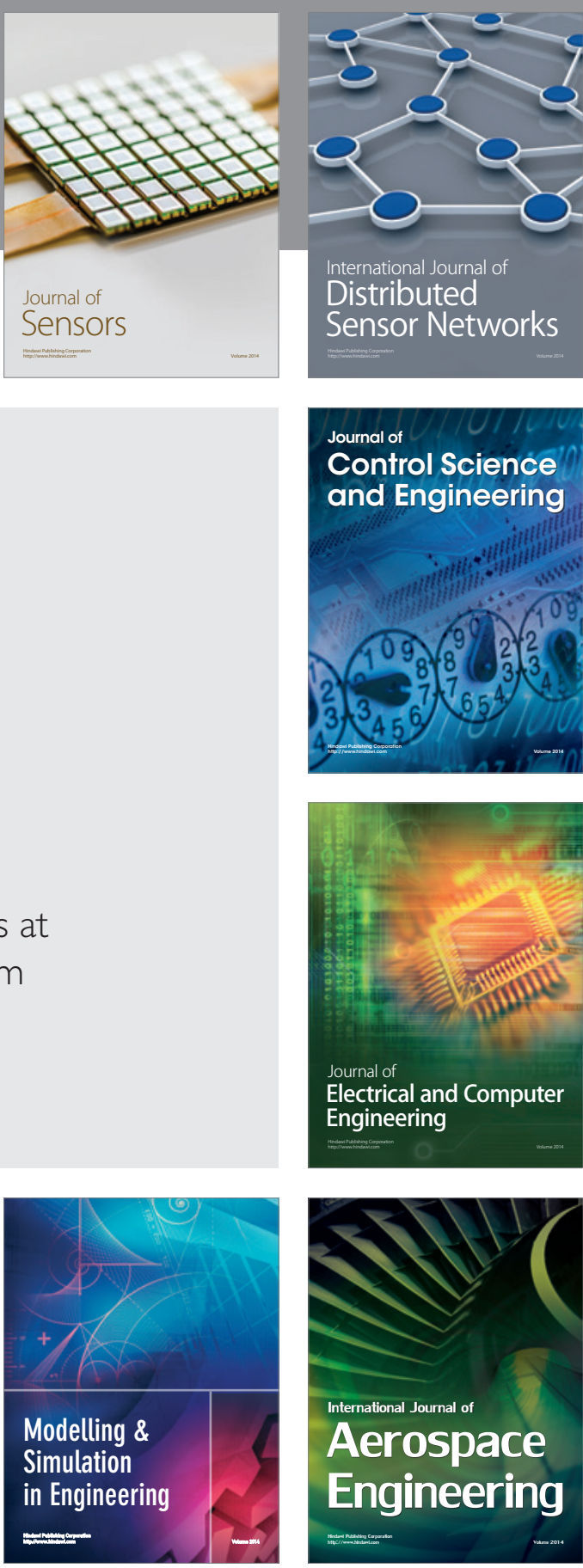

International Journal of

Distributed

Sensor Networks

Journal of

Control Science

and Engineering
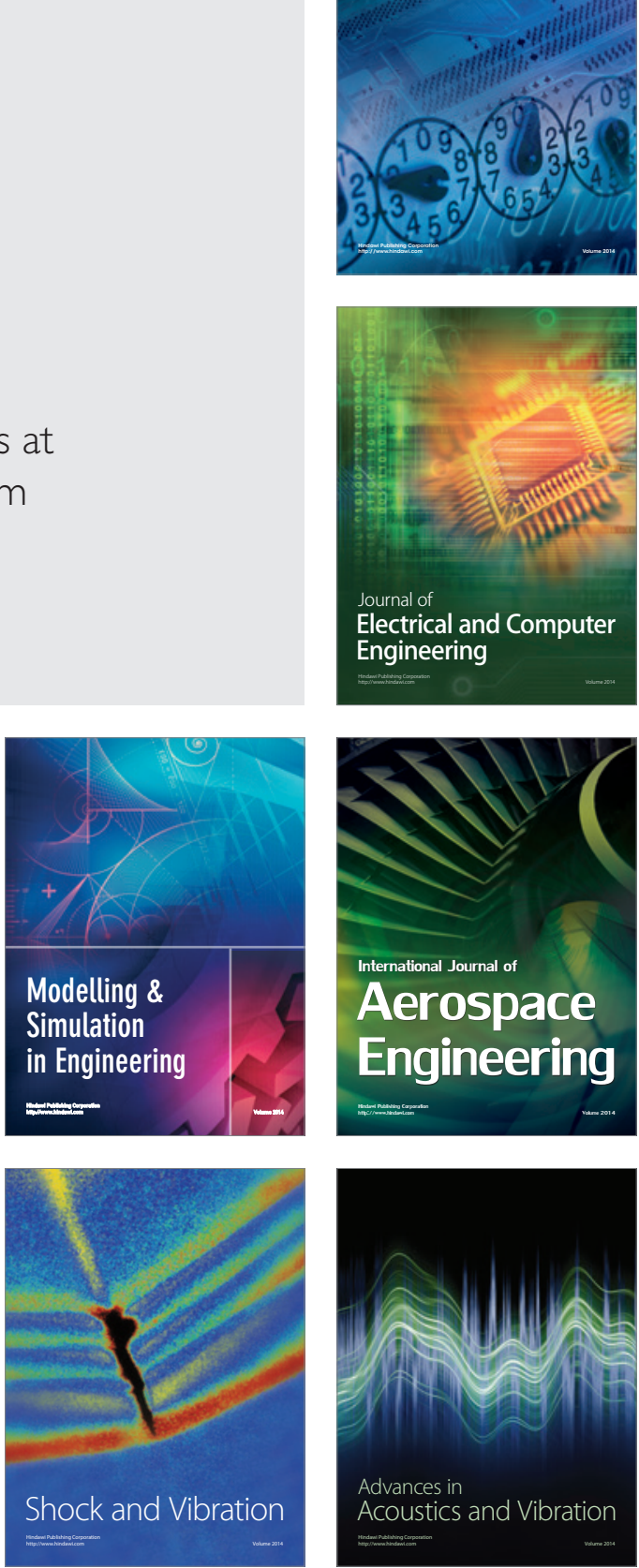